\title{
Rethinking Infrastructure Resilience Assessment with Human Sentiment Reactions on Social Media in Disasters
}

\author{
Chao Fan \\ Texas A\&M University \\ chfan@,tamu.edu
}

\author{
Hamed Farahmend \\ Texas A\&M University \\ hamedfarahmand@tamu.edu
}

\author{
Ali Mostafavi \\ Texas A\&M University \\ amostafavi@civil.tamu.edu
}

\begin{abstract}
The objective of this study is to propose and test a theoretical framework which integrates the human sentiment reactions on social media in disasters into infrastructure resilience assessment. Infrastructure resilience assessment is important for reducing adverse consequences of infrastructure failures and promoting human well-being in natural disasters. Integrating societal impacts of infrastructure disruptions can enable a better understanding of infrastructure performance in disasters and human capacities under the stress of disruptions. However, the consideration of societal impacts of infrastructure disruptions is limited in existing studies for infrastructure resilience assessment. That is because an integrative theoretical framework for connecting the societal impacts to infrastructure resilience is missing. This study proposed a theoretical framework to examine the relationship between the societal impacts and infrastructure performance in disasters using social media data. Sentiments of human messages related to infrastructure systems are adopted as an indicator of societal impacts of infrastructure disruptions. A case study for electricity and transportation systems in Houston during the 2017 Hurricane Harvey was conducted to illustrate the application of the proposed framework. We find a relation between human sentiment and infrastructure status and validate it by comparing situational information from relevant tweets with official public sources. The findings enable a better understanding of societal expectations and collective sentiments regarding the infrastructure disruptions and improve the visibility of infrastructure performance in Smart City Digital Twin in the context of disasters.
\end{abstract}

\section{Introduction}

In smart cities, infrastructure systems such as power grid, road network, and hospitals are the backbone, functioning for delivering essential services and promoting human well-being [1]. Failure of the infrastructure systems, such as power outages, pipeline damages, and road closure, is not only costly in terms of recovery, but more importantly, threatens human lives in disasters [2]. Hence, building resilient infrastructure systems is important to promote the well-being of inhabitants facing natural disasters.

Assessing and enhancing infrastructure resilience aims at reducing the failure probability of infrastructure systems and their components, minimizing severe consequences of probable failures, and increasing the pace of recovery activities following failures and losses [3]. Most of the existing studies focus on the mitigation phase of disasters, which is concerned with infrastructure vulnerability and interdependencies that increase the complexity and uncertainty of such systems and result in the emergent of unprecedented behaviors in the functionality of the systems [4]. Recently, studies of infrastructure resilience also put weight on the effectiveness of response and recovery activities to minimize disaster consequences as well as the social dimension in disaster response and recovery [5].

Despite the advances in infrastructure resilience, existing frameworks define and assess resilience mainly based on the capabilities of the built environment, and the consideration of societal impacts of infrastructure disruptions is missing. With the emergence of Smart City Digital Twin paradigm to achieve smart and resilient cities, infrastructure resilience needs to integrate societal aspects to enable more visibility and better insight in hazard mitigation and emergency response for infrastructure systems [6]. Societal impacts in the context of disasters encompass the well-being of vulnerable population regarding the damages and threats to their private properties and individual lives [7]. Integrating the societal impacts of disruptions into infrastructure resilience assessment not only provides empirical evidence of infrastructure performance in disasters, but also enables an understanding of human mental and physical capacities under the stress of infrastructure disruptions [8].

Although researchers raise the importance of societal impacts in infrastructure resilience assessment, quantifying the societal impacts in disaster disruptions is still challenging. Human sentiment, as an indicator of well-being, arises to be an effective measurement of societal impacts of disaster disruptions [9]. Recent studies have demonstrated the relationships between human sentiment and the societal impacts of disasters. For example, Baylis investigated the weather impacts using human sentiments and identified the strong 
correlation between meteorological conditions and the sentiment of human expressions [10]. Furthermore, as the use of social media like Twitter, Facebook, and Instagram is increasing, massive online social posts on these platforms become a valuable source of information to characterize human sentiments and reactions regarding infrastructure disruptions in disasters [11]. In particular, Twitter as a microblogging platform allows people to specify their situation by posting and sharing a message with up to 280 characters. Unlike traditional media such as broadcast and news articles, the participation of a great number of users enables people to efficiently communicate information, a feeling, or a reaction regarding infrastructure disruptions in disasters. Such extensive involvement and efficiency enable Twitter data to capture the human sentiments in disaster disruptions.

As society face the benefits and potential of Twitter, the studies using social media data for examining human sentiment reactions in disasters is manifold and growing rapidly, including studies that use social media data for disaster damage assessment, and relief needs classification [12]. For example, Kryvasheyeu et al. studied the spatiotemporal distribution of disasterrelated tweets and found a strong relationship between the proximities to the disaster path and the sentiments of relevant tweets [13]. Lu et al. proposed a visual analytics model for analyzing the geographical patterns of human sentiment in disasters using geotagged tweets [14].

While the analysis of human sentiments from social media data in disaster contexts is growing, little is known regarding the relation between infrastructure resilience and human sentiment reactions. One reason is that an integrative theoretical framework for connecting human sentiment reactions to infrastructure performance is missing in existing studies. To address this knowledge gap, this paper proposed a novel assessment framework for infrastructure resilience considering human sentiment reactions. A case study related to infrastructure disruptions (electricity and transportation facilities) during the 2017 Hurricane Harvey over Houston area was conducted to illustrate the application and capability of the framework.

\section{Related work}

In the past few decades, researchers have proposed multiple approaches to assess the resilience of infrastructure systems facing disruptions, including empirical approaches, network-based approaches, and simulation-based approaches. Among these approaches, network-based and simulation-based techniques are more commonly adopted in existing studies. Networkbased approaches have been used to model post-disaster

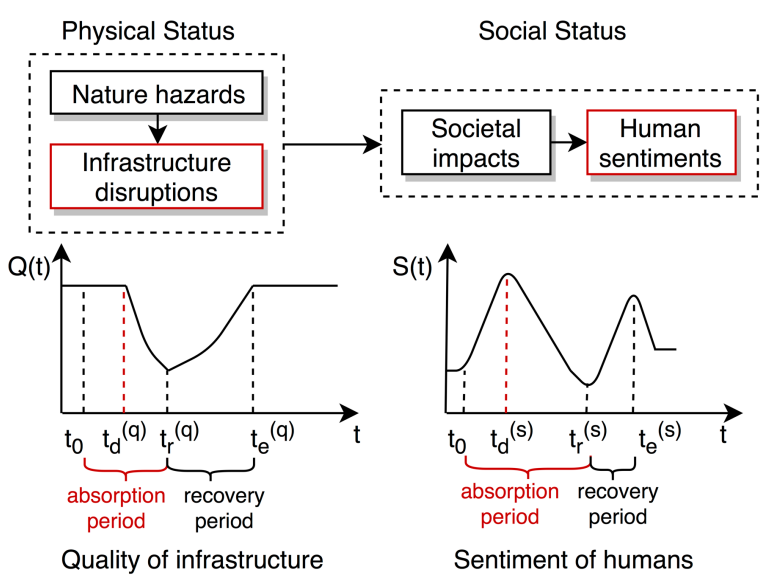

\section{Figure 1. Theoretical framework for analyzing the relationship between infrastructure resilience and human sentiments.}

functionality resilience in infrastructure systems [15]. In this models, infrastructure systems are modeled as networks to quantify resilience based on network theory and measure absorptive, adaptive, and restorative capacities [16].

In addition, simulation-based approaches employ different simulation techniques such as System Dynamics (SD) and Agent-based Modeling (ABM) to model the functionality of the system and explore system resilience. For example, SD has been used to simulate the recovery of various infrastructure systems following disasters considering uncertainties and complexities in the entire recovery process and the limitation of resources [17]. ABM is also an objectoriented simulation technique that is capable to model the performance of a system as the aggregation of the interactions of multiple entities that form the overall functionality of the system [18]. ABM contributes to integration of social behaviors and human perceptions into the risk and resilience assessment, which significantly benefits the effectiveness of disaster preparedness [19].

There is a rich body of knowledge about the infrastructure resilience assessment employing a variety of approaches and techniques to model pre-disaster preparedness, post-disaster failures and disruptions, and restoration of infrastructure systems. However, the main emphasis in such research studies is the mitigation and preparation actions and decisions to increase the capacity of the infrastructure to cope with the risk of disruptions. Thus, studies aiming at integrating societal impacts of infrastructure disruption into infrastructure resilience assessment during and after the disaster are limited. One reason is that measuring the societal impacts of infrastructure failures is also challenging in the complex disaster context. Some of the existing 
studies have employed social media data to understand the disaster impacts. For example, Samuel and Taylor studies the correlation between vulnerable populations and Twitter activities [20]. Neppalli et al. analyzed human sentiment in Hurricane Sandy using Twitter posts and showed that human sentiment varies across different locations based on the distance to disaster [21]. Chen et al. performed sentiment analysis on tweets posted during Hurricane Harvey to investigate spatiotemporal parameters impacting human sentiment in disaster situation [22].

In sum, since the infrastructure systems play key roles in maintaining the well-being of the residents at risk and developing opportunities to promote the quality of life in the community, consideration of the societal impact of the infrastructure disruption in the assessment of resilience is imperative. Therefore, a proper resilience assessment framework should be able to consider the interconnection between the human and infrastructure. The framework should also be able to appropriately deal with large-scale human-generated data in infrastructure disruptions.

\section{The proposed theoretical framework}

During the last decades, multiple resilience models have been proposed to characterize the dynamic situation and measure the quality of infrastructure systems under the stress of disasters. For generality, the quality of infrastructure is assumed to represent the abilities of the infrastructure that can provide functioning services to the residents [23]. The first conceptual model was proposed by Bruneau et al., which measures the resilience of infrastructure as the size of the expected degradation in quality (probability of failure), over time (that is, time to recovery) [3]. The proposed theoretical framework for infrastructure resilience assessment is built upon this basic idea and integrate the connections between the reactions of vulnerable population and the performance of the built environment which are exposed to the natural hazards (see Figure 1).

\subsection{Definitions of time and periods}

To establish a common framework for examining infrastructure resilience with the consideration of human sentiments, a unified terminology is proposed, while their relationships are analyzed and presented in this paper.

Built environment including infrastructure systems is firstly attacked by disasters when the disasters approach the urban area (Figure $1, t_{0}$ ). With the increase of the intensity and the cumulative stresses of the natural disasters, disruptions start appearing in the built environment (Figure $1, t_{d}^{(q)}$ ). The disruptive events such as utility cutoff and road closure further make negative effects on human's lives including limited access to grocery stores and not being able to cook. The quality of the infrastructure systems reaches to the minimum at time $t_{r}^{(q)}$ [6]. Affected people have to take response actions to protect themselves, repair the damages, restore the qualities of infrastructure systems, and resist the stress of natural disasters. After disasters pass and restoration efforts are conducted, the infrastructure systems resume operations at time $t_{e}^{(q)}$, and people's sentiment also goes up again.

There are two important periods for measuring infrastructure resilience and human sentiments: absorption period and restoration period. In the absorption period, infrastructure systems absorb the stress from natural disasters. For example, the stress of regular weather conditions such as drizzle and breeze can be absorbed by the infrastructure systems and will not lead to any disruptions. However, the infrastructure cannot withstand extreme weather conditions such as hurricanes, snowstorms, and earthquakes because the stress of extreme weather conditions exceeds the absorptive capacities of the infrastructure systems. Physical disruptions serve as a form of absorption for infrastructure to absorb the stress from the extreme weather. The quality of the infrastructure drops to the nadir when the physical disruptions happen. Hence, we define the absorption period for infrastructure systems is the time period between $t_{0}$ and $t_{r}^{(q)}$. In the restoration period, the infrastructure systems restore their functioning based on the response and recovery actions and the unloading of disasters. Thus, the recovery period is defined as the period between $t_{r}^{(q)}$ and $t_{e}^{(q)}$. Coupling these two periods (from $t_{0}$ to $t_{e}^{(q)}$ ), the resilience of infrastructure systems tends to be measured by a resilience triangle implying the absorptive capacity and restorative capacity of the infrastructure.

Facing the disasters, people may have their own expectations for the intensity of the disasters and the damages that would be caused by disasters. As affected people experience the disruptive events and the subsequent adverse impacts (e.g., life loss and property damages), they would have different sentiments regarding the differences between the real disruption situation and their expectations. That is, people would have highly positive expressions when the real situation is better than their expectations at time $t_{d}^{(s)}$. For example, there are fewer damages or closed road sections than the affected people predicted before the disruptions. On the contrary, the sentiment of the human expressions would drop fast if the severity of the 
disruptions exceeds the expectations and the affordable capacities of affected people and reach to the minimum at time $t_{r}^{(s)}$. Similarly, the absorption period for humans is defined as the period between $t_{0}$ and $t_{r}^{(s)}$. Finally, the human sentiment goes up when the services are restored after the disasters at time $t_{e}^{(s)}$. The recovery period for humans is defined as the period between $t_{r}^{(s)}$ and $t_{e}^{(s)}$.

\subsection{Formulations of temporal differences}

The dynamics of the infrastructure quality is corresponding to the human sentiment in their relevant posts, which can indicate the impacts of the infrastructure performance on human lives. The changes in human sentiments are also correlated to the timing of the infrastructure disruptions and restoration. However, the periods for infrastructure and humans do not always perfectly align. Due the expectations and affordable capacities of affected people, there exists temporal differences of the time and periods between infrastructure and humans. To better characterize their correspondence and relations, we formulate two indicators to quantify the temporal differences.

The first temporal difference captured in the framework is the difference between the time $t_{d}^{(q)}$ when infrastructure disruption happens and the time $t_{d}^{(s)}$ when human sentiment starts to drop. We define this difference, $\Delta_{d}$, as the capacity of humans to afford the adverse impacts of the infrastructure disruptions. The larger the $\Delta_{d}$ is, the higher the capacity of the affected people to withstand the impacts of disruptions.

$$
\Delta_{d}=\left|t_{d}^{(q)}-t_{d}^{(s)}\right|
$$

Similarly, we can define $\Delta_{r}$ as the difference between the time $t_{r}^{(q)}$ that disruption restoration is triggered and the time $t_{r}^{(s)}$ that the human sentiment reaches to minimum and starts to raise. $\Delta_{r}$ is to measure the extent to which the performance of infrastructure recovery satisfies the expectations of the humans and the extent to which the recovery of infrastructure relieves the negative impacts of the disruptions.

$$
\Delta_{r}=\left|t_{r}^{(q)}-t_{r}^{(s)}\right|
$$

This indicator is important to measure the response and recovery performance of facility agencies regarding the service disruption. The higher the $\Delta_{r}$ is, the worse the recovery is. These two quantitative indicators are able to capture the relation between infrastructure performance and human sentiments, which contribute to a comprehensive examination of infrastructure resilience to disasters.

\subsection{Model verification and validation}

Once the dynamics of infrastructure performance and human sentiments are characterized by the proposed framework. There remains a critical question, whether collective human sentiment exactly indicate humans' perceptions regarding the performance of infrastructure. This question is important because it is the key to the reliability and validity of the results and finding generated by the proposed framework. In this framework, we employed two steps to conduct the model verification and validation to ensure the validity and reliability of the results and findings.

First, to verify the reliability and credibility of the process when implementing the framework in real cases, we proposed two approaches: verifying the content of the tweets to confirm that the content and the sentiment are matched; and verifying the user distribution of their sentiments to confirm that there is no dominant users who govern the collective sentiments. These two approaches can benefit the reliability of the sentiment measurement and the findings when comparing the sentiments and infrastructure performance.

Second, we validate the findings and implications by using external sources such as public data from infrastructure agencies, and household surveys from the disaster-affected area. The data from infrastructure agencies can precisely record the status of the infrastructure facilities during disasters and have quantitative measurements to indicate the performance of service facilities. Additionally, household survey is an important approach to collect the data about human perceptions, impacts and reactions during the disasters. Hence, the results from the household survey is reliable to validate the results of the human sentiments analyzed by the proposed framework.

\section{Case study of Hurricane Harvey}

To illustrate the capabilities of the proposed theoretical framework, we conducted a case study for two severe infrastructure disruptions (i.e., power outages, and road closure) during the 2017 Hurricane Harvey.

\subsection{Disaster context and data collection}

Hurricane Harvey, a category 4 tropical storm, landed in Houston late August in 2017. As shown in Figure 2(a), Hurricane Harvey brought rainfall from August 27 to August 29, caused large-scale flooding and undermined infrastructure systems in the affected areas. 
Table 1. Filtering keywords and the sizes of the relevant data

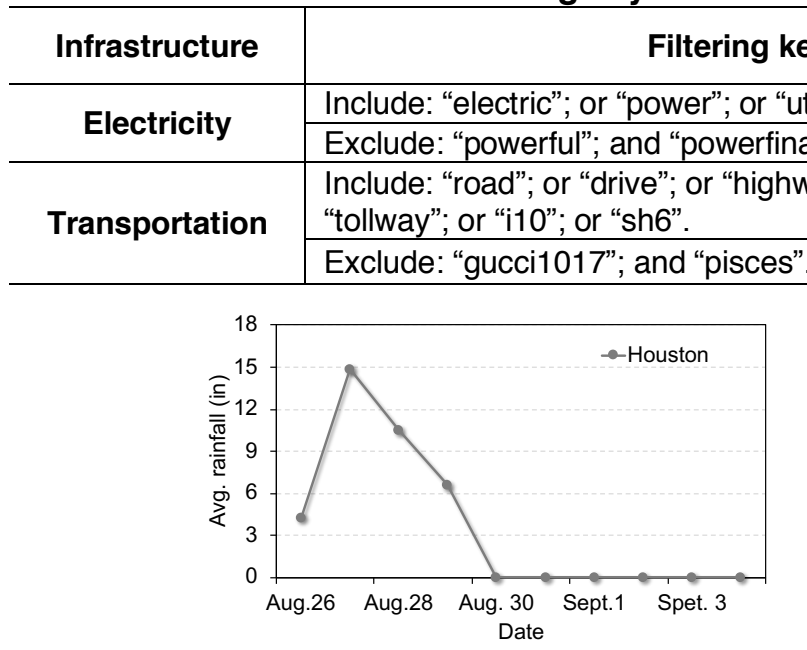

(a)

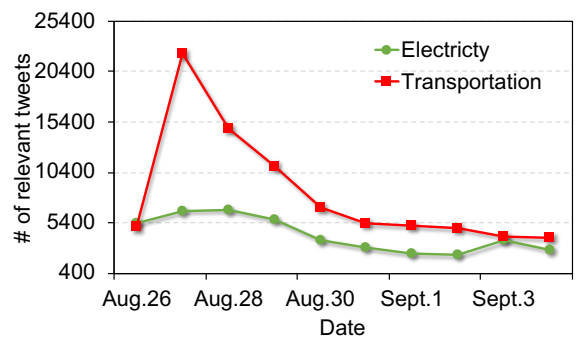

(b)

Figure 2. Average rainfall in Houston (a) and number of tweets related to the infrastructure (b).

Specifically, more than 200 road sections were closed due to flooding, and more than 100 thousand residents are experiencing power outage due to the devastation of transmission cables [24]. As such, power systems (a.k.a., electricity) and transportation systems are two of the most severely affected infrastructure systems during Hurricane Harvey.

To investigate the impacts of Hurricane Harvey during its entire life cycle, we collected the tweets generated by Houston residents before, during and after Hurricane Harvey (from August 22 to September 30). Twitter Power Track API allows us to define the rules based on the geographical information in the tweets, and the user localities in their profiles [25]. Thus, to have a complete picture of how humans behave in response to the disaster, we define bounding boxes to cover the entire Houston area, and also gather the tweets posted by the users whose profile locality is in Houston. Using these two criteria, we finally collected about 21 million tweets for studying human reactions on social media in the context of Hurricane Harvey.

To specifically examine the performance and human sentiments for electricity and transportation systems, we specified some relevant keywords to filter the tweets from our complete dataset (see Table 1). Meanwhile, using keywords for searching tweets may also induce some noises in which the tweets contain the keywords, but the content is irrelevant. To minimize the induced noises, we went through the unique tweets in the filtered datasets, identified some high-frequency noises which are distinguishable by some irrelevant keywords, and excluded these noises by adding rules in the filtering process. In addition, because we mainly focus on the performance and the societal impacts of the infrastructure systems during and a few days after the disaster, we select the data one day before the disaster (August 26), three days during the disasters (from August 27 to August 29), and six days after the disaster (from August 30 to September 4).

Using both inclusion and exclusion keywords and the time period, we finally get more than 41 thousand tweets related to electricity systems and more than 83 thousand related to transportation systems. Figure 2(b) shows the number of relevant tweets generated by resident users each day during Hurricane Harvey. In general, the numbers of relevant tweets have similar trends as the intensity of the rainfall when Hurricane Harvey approached to Houston. However, the tweets related to transportation systems had quadrupled in the first day of the disaster, which indicates damages and human concerns related to transportation systems. The following sections will measure the human sentiments in these relevant tweets and analyze the relationship between infrastructure resilience and human sentiments.

\subsection{Human sentiments on social media}

Detecting sentiments for social media messages is an important component in the proposed framework. Human sentiments can be either categorical (e.g., positive, negative or neutral) or numerical (e.g., scores for representing the sentiment) [26]. With the development of sentiment analysis in the past decades, the methods for labeling the sentiments for texts are mainly in two types: supervised learning methods and lexicon-based methods. Supervised learning methods such as Support Vector Machine, Naive Bayes, and Maximum Entropy algorithms require labeling, training, and validation for detecting the sentiment for a set of data. Lexicon-based methods such as VADER 


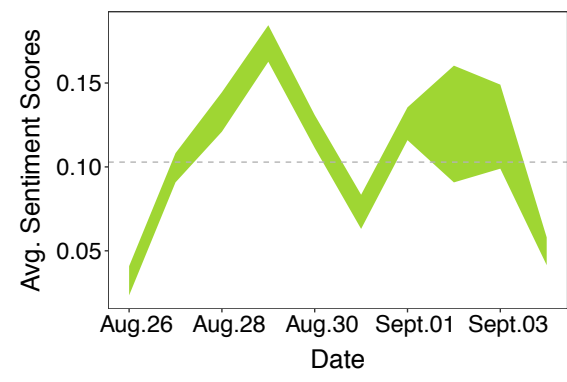

(a)

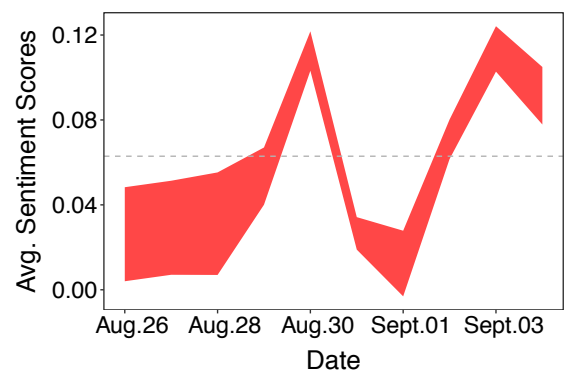

(b)

Figure 3. Average sentiment scores of human reactions related to (a) electricity and (b) transportation on social media during Hurricane Harvey. The width of the line in both figures depends on the slope of the line. The lower the slope, the wider the line.

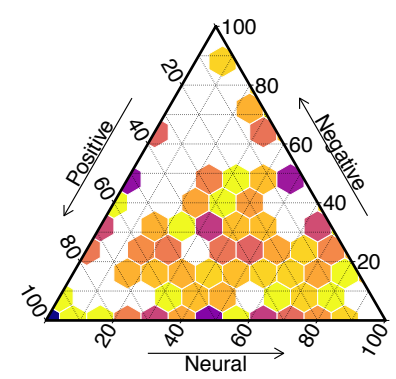

(a)

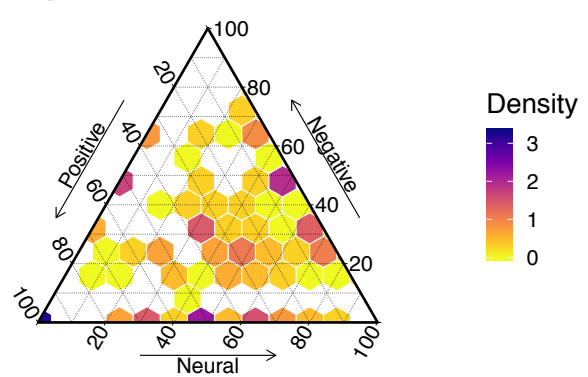

(b)

Figure 4. The distribution of sentiment population on social media during (a) absorptive period (Aug.28 - Aug. 29) and (b) restorative period (Aug. 30 - Aug. 31). The density of the population in each diamond is normalized by the ratio of the population in two periods.

(Valence Aware Dictionary for sEntiment Reasoning) combine the dictionary of emotions where words are attributed to a given sentiment strength and predefined rules for classifying sentiments [26]. Recent studies tested both lexicon-based methods and supervised learning methods on Twitter data, and the lexicon-based methods show the high quality in the results.

The accuracy of VADER in Twitter data for hurricane context has been demonstrated in the study for Hurricane Sandy [27]. As such, this study employed the VADER Sentiment package, a lexicon and rule-based sentiment analysis library considering also lexical features of tweets such as sentiment-related acronyms and initialisms (e.g., LOL and WTF) [28]. VADER gives the sentiment scores between -1 (extremely negative sentiment) and +1 (extremely positive sentiment), with 0 values representing sentiment neutrality. To capture the collective sentiment across all social media activities each day, we averaged the sentiment scores for all relevant tweets generated each day and mapped the changes in the 10-day period.

As shown in Figure 3, the average sentiment scores for electricity system and transportation system have a similar trend. Starting from neutrality, the average sentiment scores went up first. It indicates that the infrastructure systems perform better than people's expectation, even though disruptions had happened at that time. However, the infrastructure systems reached the maximum of their absorptive capacity fast and broke down, which caused significant adverse impacts on affected people. Thus, human sentiments in relevant tweets declined rapidly in both figures. Then, after Hurricane Harvey dissipated and response actions such as maintenance applied, partial restoration of failure infrastructure systems make the services recovered. Human sentiments on social media were also dominated by highly positive messages when the infrastructure restarted functioning and then returned to neutrality. The inferences and findings from the results in this section will be validated in the following two sections.

Further, to characterize the sentiment of online users during the disasters, we need to convert the sentiment scores into categories for the tweets. As mentioned earlier, the sentiment scores detected using VADER approach ranges from -1 to +1 . We divided the scale of the score into three sections and classified the tweets into positive (from +0.33 to +1 ), neural (from 0.33 to +0.33 ), and negative (from -1 to -0.33 ) categories based on their sentiment scores. Then, we calculated the ratio of the tweets in three categories for all online users. Figure 4 shows the population distribution with different ratios of positive, negative, and neutral tweets in absorption and restoration periods. Comparing the results in these two figures, we can find 
Table 2. Infrastructure status carried by human sentiment reactions on social media

\begin{tabular}{|c|c|c|c|}
\hline Infrastructure & Date & Sentiment & Human Sentiment Reactions on Twitter \\
\hline \multirow{9}{*}{ Electricity } & Aug. 26 & Negative & $\begin{array}{l}\text { "Downed poles \&amp;amp; power lines in the } 3300 \text { block of Ave I. } \\
\text { Roadway is shut down in the area. Harvey." }\end{array}$ \\
\hline & Aug. 27 & Negative & $\begin{array}{l}\text { "It's all good til the storm knock the power out right b4 the damn fight. } \\
\text { Fuck you, Harvey." }\end{array}$ \\
\hline & Aug. 28 & Positive & $\begin{array}{l}\text { "My house didn't flood, the power didn't go out and we have plenty of } \\
\text { water I feel so blessed GOD IS GOOD!!!" }\end{array}$ \\
\hline & Aug. 29 & Positive & $\begin{array}{l}\text { "House didn't flood, cars didn't flood, didn't lose electricity even once. } \\
\text { My family \&amp; neighbors are so fortunate, beyond the..." }\end{array}$ \\
\hline & Aug. 30 & \multirow{2}{*}{ Negative } & \multirow{2}{*}{$\begin{array}{l}\text { "houstonisd may not open Sept. 5. They are still evaluating. Some } \\
\text { schools have power issues, roof damage..." }\end{array}$} \\
\hline & Aug. 31 & & \\
\hline & Sept. 1 & \multirow{2}{*}{ Positive } & \multirow{2}{*}{$\begin{array}{l}\text { "Good Evening. If you are still without power, contact us with your } \\
\text { service address in a DM so I can investigate. Thanks." }\end{array}$} \\
\hline & Sept. 2 & & \\
\hline & Sept. 3 & Positive & "Power is so mf good" \\
\hline
\end{tabular}

that fewer people in the absorption period posted negative tweets, while the majority of the people in the restoration period have a large proportion of negative tweets. The results of the population's sentiments are consistent with the results for all relevant tweets. The results also imply that the average sentiment scores are derived from the affected population and not driven by some dominant Twitter accounts.

\subsection{Graph-based text summarization}

A large number of relevant social media data were generated by disaster-affected people during the disaster. However, going through all relevant tweets to summarize the important situational information is time-consuming. As suggested by existing studies, important situational information tends to be repeated and retweeted many times on social media [29]. Accordingly, the tweets which contain important situational information will have high similarities with the other relevant tweets and also have lots of retweets. Based on these assumptions, a graph-based approach was adopted to capture critical situational information related to the infrastructure systems by identifying critical relevant tweets [25], [30].

First, all relevant tweets for an infrastructure system were represented by a vector using a latent space model, term-frequency-inverse-document frequency (tf-idf). Then, we calculated the pairwise cosine similarities among all tweet vectors. Doing so, we can construct a weighted semantic graph, $G=(V(G), E(G), w)$, in which the nodes represent the tweet vectors, the edges and the weights represent the similarities among the tweet vectors. Using the degree centrality in network theory, we can calculate the weighted degree centrality for each node and rank them to identify the critical tweets which have the highest degree centrality in the graph. Finally, we converted the critical tweet vectors back to the original tweets for reviewing the situational information contained in the tweets.

Table 2 shows the results of the graph-based text summarization for the situation related to the electricity systems during and after disasters. The sentiment categories labeled by the VADER package are also displayed for each critical tweet. The text summarization for transportation systems shows the same findings. To avoid redundancy, here, we only show the results for electricity systems. As implied by the results, the power outage caused by the disasters would lead to negative sentiment for affected people (situation on August 27). However, people would also have highly positive sentiment if the performance of the infrastructure exceeds their expectation (situation on August 28). When Hurricane Harvey dissipates, the power system was repaired in time and gradually restored. Therefore, the messages with highly positive sentiments on social media were growing and becoming dominant (situation on September 2 and 3). The results in Table 2 show that the human sentiment on social media is corresponding to the infrastructure status. Such relationship provides useful insights allowing us to assess the infrastructure resilience through consideration of human sentiments on social media.

\subsection{Framework calibration and validation}

To validate the relation between human sentiments in relevant tweets and the infrastructure performance during Hurricane Harvey, we collected statistical data from infrastructure management agencies to make a comparison between average sentiment scores on social media and disruptive events in the built environment. 


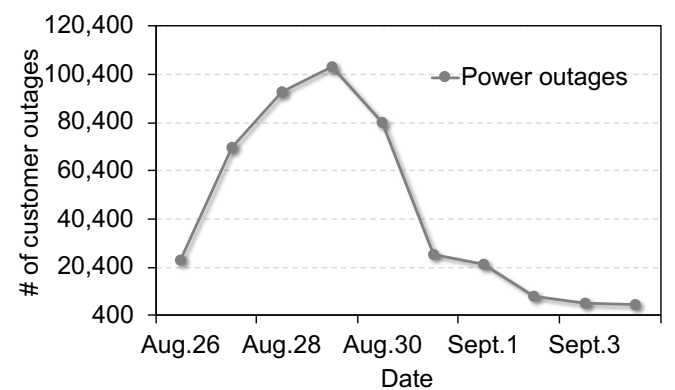

(a)

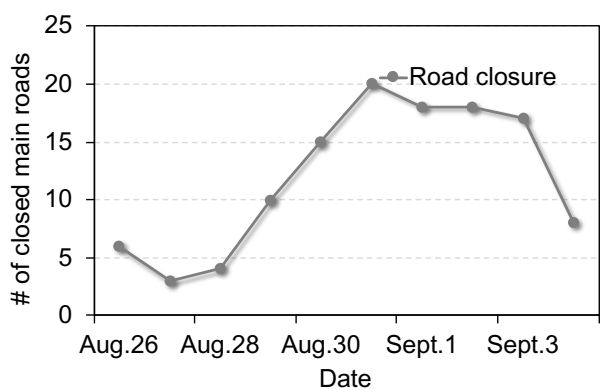

(b)

Figure 5. Number of customer outages of CenterPoint Energy (a) and number of closed main roads (b) in Houston during Hurricane Harvey.

The functioning of electricity systems mainly replies on the quality of the transmission and distribution service providers (TDSPs). In Houston, CenterPoint Energy serves as the area's TDSP and works with about 85 Retail electric providers (REPs) [31]. Thus, instead of customers' own REP, CenterPoint Energy takes the responsibility of the power services in Houston. Accordingly, we collected the data for the number of customers outages during Hurricane Harvey from the U.S. Department of Energy to examine the performance of the electricity systems in disasters (see Figure 5(a)) [32]. The number of power outages increased fast at the beginning of the disaster. That is a common situation in a lot of previous disasters [33]. Thus, the cutoff of the electricity did not lead to highly negative sentiments on social media. On the contrary, human sentiment increased because some areas that had experienced power outage in previous disasters had power during Hurricane Harvey. The performance of the power systems exceeds the expectations of the people in the affected area. However, when the power outage continuously expanded and persisted, people's sentiments on social media declined fast. Since the restoration of electricity systems was very efficient after Hurricane Harvey, human sentiment returned to growth.

To validate the results related to transportation systems, we gather the data about the closed roads in Houston from a partner of the City of Houston [34]. There are about 187 closure reports with the start and end dates of closure for the main roads in Houston. The period of a road closure includes the dates when the closure starts and ends. As shown in Figure 5(b), the number of closed roads increased along with the cumulative stress of Hurricane Harvey. During the first three days, the transportation systems absorbed most of the stress from the disaster, which leads to an increase in human sentiments on social media. However, in the following four days, the number of closed roads kept increasing and reached to a peak point on August 31. Hence, human sentiments on social media declined significantly and reached to the lowest point on August

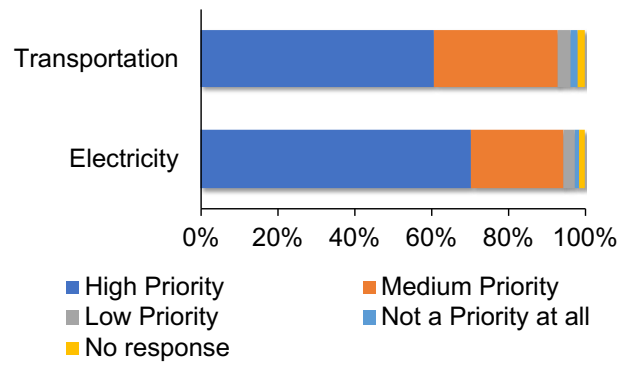

Figure 6. Residents Attitude about Priority for Investment in Transportation and Electricity systems

31 as well. After the flood water receded, the number of closed roads decreased and the human sentiment correspondingly increased. Based on the observations, we find that the dynamics of human sentiments in social media activities correlate to the performance of the infrastructure systems during the disasters.

Considering the time that Hurricane Harvey approaches the area $\left(t_{0}\right)$ as $t_{d}^{(q)}$ for both infrastructure systems, we can define and measure $\Delta_{d}$ measuring the difference between the infrastructure disruption start time $t_{d}^{(q)}$ and the time sentiment start to drop $t_{d}^{(s)}$, which indicates that the disruption is exceeding absorption capacity. Results show that $\Delta t_{a}$ for electricity is 3 days while for transportation it is 4 days. It indicates that the disruption in transportation has been more tolerable and/or expected for residents. Similarly, we can define and measure $\Delta t_{r}$, which is the difference between the time that disruption restoration is triggered $t_{r}^{(q)}$ and the time that the corresponding human sentiment reaches to minimum and starts to raise $t_{r}^{(s)}$. For the case of Hurricane Harvey in the study area, $\Delta t_{r}$ for electricity is equal 2 days while it equals 1 day for transportation. It indicates that the restoration of electricity has not been in accordance with human expectation compare to the restoration of transportation system. To better understand this phenomenon, we compared the findings of this study with a result of a household survey conducted by the authors following 
Hurricane Harvey which investigates residents' attitude regarding investment priorities for infrastructure systems during recovery period of Harvey. The survey asks 209 individuals about their perceived priority of investment in different infrastructure systems. Figure 6 compares residents' attitudes regarding priority of investment in Transportation and Electricity. It can be seen that overall, residents support putting higher priority on electricity. It is aligned with the finding there is a need to invest to make electricity more resilient to meet the expectations of residents in future floods.

\section{Concluding Remarks}

This paper proposed and tested a theoretical framework to integrate societal impacts of infrastructure disruptions for infrastructure resilience assessment. The application of the proposed framework was demonstrated in a case study of electricity and transportation systems in Houston during the 2017 Hurricane Harvey. The results show that the dynamics of human sentiments in relevant social media activities correlate to the performance of infrastructure systems in disasters. The findings contribute to a better understanding of infrastructure resilience and human mental and physical capacities under the stress of disaster disruptions. Assessing infrastructure resilience is inevitably a complex process in which human and infrastructure are dynamically involved and interact with each other. This study is a first attempt for structuring the complex relationships between human and infrastructure systems in disasters. The proposed framework can be generalized and integrated in the Smart City Digital Twin paradigm to enable monitoring and analysis of the performance of infrastructure in disasters. As such, the digital twin can not only have the digital version of physical facilities and environment, but also incorporate the digital version of humans in coping with the disruptions. Through the use of digital twin, in particularly, future studies can examine other disaster contexts and evaluate the universality of the human-infrastructure relation identified in case study.

Practically, the digital twin of smart cities incorporating human sentiment can allow infrastructure management agencies to better capture and respond to the disruptive events. The relationship between human sentiments and the infrastructure status can imply the capacities of vulnerable people to withstand the adverse impacts of the disasters. For example, the fact that the sentiment about electricity starts to drop in 3 days after the disruption shows that there is a need to plan to enhance the resilience in power system to avoid power outage for more than 3 days. Besides, the pace of restoration for electricity has not been aligned with residents' expectations, which should be considered in resilience enhancement and risk mitigation programs. In addition, the findings also provide evidence for prioritization of response and recovery actions. For example, as discussed in framework calibration section, the effects of power outages and road closures vary. People's endurance for the failure of transportation is more than their capacity for power outages in the studied case. Considering other evidences such as infrastructure interdependencies in the Smart City Digital Twin, the infrastructure management agencies can make better decisions on the allocation of their response and recovery efforts.

Although the proposed theoretical framework can capture empirical evidence for assessing infrastructure resilience, there are also some induced limitations. The most important limitation is that, human sentiments are measured from empirical datasets in past natural disasters and difficult to be incorporated in simulation. The smart city digital twin has a component of simulation to assess and examine the resilience capacities for optimized infrastructure systems. Further studies can extend this framework to quantify people's expectations for the performance of infrastructure systems in disasters and deal with the potential attacks such as cyber-attacks in digital twin.

\section{Acknowledgements}

This material is based in part upon work supported by the National Science Foundation under Grant Number IIS-1759537, CMMI-1832662 and the Amazon Web Services (AWS) Machine Learning Award. Any opinions, findings, and conclusion or recommendations expressed in this material are those of the authors and do not necessarily reflect the views of the National Science Foundation and Amazon Web Services (AWS).

\section{References}

[1] M. Ouyang and Y. Fang, "A Mathematical Framework to Optimize Critical Infrastructure Resilience against Intentional Attacks," Comput. Civ. Infrastruct. Eng., vol. 32, no. 11, pp. 909-929, 2017.

[2] D. Singh, M. Tsiang, B. Rajaratnam, and N. S. Diffenbaugh, "Observed changes in extreme wet and dry spells during the south Asian summer monsoon season," Nat. Clim. Chang., 2014.

[3] M. Bruneau et al., "A Framework to Quantitatively Assess and Enhance the Seismic Resilience of Communities," Earthq. Spectra, vol. 19, no. 4, pp. 733-752, Nov. 2003.

[4] A. A. Ghorbani and E. Bagheri, "The state of the art in critical infrastructure protection: a framework for convergence," Int. J. Crit. Infrastructures, vol. 4, no. 3, p. 215 , 2008 . 
[5] S. Chang, "Infrastructure resilience to disasters," Bridg., vol. 44, no. 3, 2014.

[6] N. Mohammadi and J. E. Taylor, "Smart City Digital Twins," in IEEE Symposium Series on Computational Intelligence (SSCI), 2017, pp. 1-5.

[7] K. Brown and E. Westaway, "Agency, Capacity, and Resilience to Environmental Change: Lessons from Human Development, Well-Being, and Disasters," Annu. Rev. Environ. Resour., vol. 36, no. 1, pp. 321-342, Nov. 2011.

[8] A. Mostafavi, D. Abraham, and A. Vives, "Exploratory analysis of public perceptions of innovative financing for infrastructure systems in the U.S.," Transp. Res. Part A Policy Pract., vol. 70, pp. 10-23, 2014.

[9] A. Steptoe, J. Wardle, and M. Marmot, "Positive affect and health-related neuroendocrine, cardiovascular, and inflammatory processes.," Proc. Natl. Acad. Sci. U. S. A., vol. 102, no. 18, pp. 6508-12, May 2005.

[10] P. Baylis et al., "Weather impacts expressed sentiment," PLoS One, vol. 13, no. 4, p. e0195750, Apr. 2018.

[11] C. Zhang, C. Fan, W. Yao, X. Hu, and A. Mostafavi, "Social media for intelligent public information and warning in disasters: An interdisciplinary review," International Journal of Information Management. 2019.

[12] J. R. Ragini, P. M. R. Anand, and V. Bhaskar, "Big data analytics for disaster response and recovery through sentiment analysis," Int. J. Inf. Manage., vol. 42, no. May, pp. 13-24, 2018.

[13] Y. Kryvasheyeu et al., "Rapid assessment of disaster damage using social media activity," $S c i$. $A d v$., vol. 2, no. 3, 2016.

[14] Y. Lu, X. Hu, F. Wang, S. Kumar, H. Liu, and R. Maciejewski, "Visualizing Social Media Sentiment in Disaster Scenarios," in Proceedings of the 24th International Conference on World Wide Web - WWW'15 Companion, 2015, pp. 1211-1215.

[15] Y.-P. Fang, N. Pedroni, and E. Zio, "Resilience-Based Component Importance Measures for Critical Infrastructure Network Systems," IEEE Trans. Reliab., vol. 65, no. 2, pp. 502-512, Jun. 2016

[16] S. Hosseini and K. Barker, "Modeling infrastructure resilience using Bayesian networks: A case study of inland waterway ports," Comput. Ind. Eng., vol. 93, pp. 252-266, Mar. 2016.

[17] S. Hwang, M. Park, H.-S. Lee, S. Lee, and H. Kim, "Postdisaster Interdependent Built Environment Recovery Efforts and the Effects of Governmental Plans: Case Analysis Using System Dynamics," J. Constr. Eng. Manag., vol. 141, no. 3, p. 04014081, Mar. 2015.

[18] K. Rasoulkhani and A. Mostafavi, "Resilience as an emergent property of human-infrastructure dynamics: A multi-agent simulation model for characterizing regime shifts and tipping point behaviors in infrastructure systems," PLoS One, vol. 13, no. 11, p. e0207674, Nov. 2018.

[19] J. Dubbelboer, I. Nikolic, K. Jenkins, and J. Hall, “An Agent-Based Model of Flood Risk and Insurance," J. Artif. Soc. Soc. Simul., vol. 20, no. 1, p. 6, Jan. 2017.

[20] R. Samuels and J. E. Taylor, “Applied Methodology for Identifying Hurricane-Induced Social Media Signal Changes in Vulnerable Populations Rachel," in Computing in Civil
Engineering, 2019, pp. 439-446.

[21] A. Squicciarini, A. Tapia, and S. Stehle, "Sentiment analysis during Hurricane Sandy in emergency response," Int. J. Disaster Risk Reduct., vol. 21, pp. 213-222, Mar. 2017.

[22] S. Chen, J. Mao, and G. Li, "Spatiotemporal Analysis on Sentiments and Retweet Patterns of Tweets for Disasters," in Lecture Notes in Computer Science (including subseries Lecture Notes in Artificial Intelligence and Lecture Notes in Bioinformatics), 2019, vol. 11420 LNCS, pp. 436-443.

[23] C. Nan and G. Sansavini, "A quantitative method for assessing resilience of interdependent infrastructures," Reliab. Eng. Syst. Saf., vol. 157, pp. 35-53, 2017.

[24] ENR Editors, "How Badly Has Hurricane Harvey Damaged Texas Infrastructure? | 2017-08-28 | ENR," Engineering News-Record, 2017. [Online]. Available: https://www.enr.com/articles/42639-how-badly-has-

hurricane-harvey-damaged-texas-infrastructure. [Accessed: 12-Feb-2018].

[25] C. Fan, A. Mostafavi, W. Yao, and R. Huang, "A Graphbased Approach for Detecting Critical Infrastructure Disruptions on Social Media in Disasters," in 2019 52th Hawaii International Conference on System Sciences (HICSS), 2019, pp. 1975-1984.

[26] M. Stella, E. Ferrara, and M. De Domenico, "Bots increase exposure to negative and inflammatory content in online social systems.," Proc. Natl. Acad. Sci. U. S. A., vol. 115, no. 49, pp. 12435-12440, Dec. 2018.

[27] L. Zou, N. S. N. Lam, H. Cai, and Y. Qiang, "Mining Twitter Data for Improved Understanding of Disaster Resilience," Ann. Am. Assoc. Geogr., vol. 108, no. 5, pp. 1422-1441, Sep. 2018.

[28] C. J. Hutto and E. Gilbert, "VADER: A Parsimonious Rule-Based Model for Sentiment Analysis of Social Media Text," Eighth Int. AAAI Conf. Weblogs Soc. Media, May 2014. [29] C. Fan and A. Mostafavi, "A graph-based method for social sensing of infrastructure disruptions in disasters," Comput. Civ. Infrastruct. Eng., 2019.

[30] Y. Wang and J. E. Taylor, "Urban Crisis Detection Technique: A Spatial and Data Driven Approach Based on Latent Dirichlet Allocation (LDA) Topic Modeling," in Proceedings of the 2018 Construction Research Congress 2018, 2018, pp. 428-438.

[31] ChooseTexasPower, "Compare Houston Electricity Providers," ChooseTexasPower.org, 2017. [Online]. Available: https://www.choosetexaspower.org/in/

Houston/. [Accessed: 15-Jun-2019].

[32] U.S. Department of Energy, "Hurricanes Maria, Irma and Harvey Situation Reports," 2017. [Online]. Available: https://www.energy.gov/ceser/downloads/hurricanes-mariairma-and-harvey-situation-reports-archived-august-26-2017september. [Accessed: 15-Jun-2019].

[33] S. D. Guikema, R. Nateghi, S. M. Quiring, A. Staid, A. C. Reilly, and M. Gao, "Predicting Hurricane Power Outages to Support Storm Response Planning," IEEE Access, vol. 2, pp. 1364-1373, 2014.

[34] Houston Transtar, "Houston TranStar - Traffic Map," Houston Transtar, 2017. [Online]. Available: https:// traffic.houstontranstar.org/layers/. [Accessed: 15-Jun-2019]. 